\title{
Rudolf von Jhering (1818-1892) und Der Zweck im Recht (1877/1883) in den Niederlanden
}

Der Zweck ist der Schöpfer des ganzen Rechts. ${ }^{1}$

\section{Einleitung}

Jhering war in den juristischen Kreisen des 19. Jahrhunderts der Niederlande kein Unbekannter. Er hatte zum Beispiel persönlichen Kontakt zum Amsterdamer Richter S.J. Hingst (1834-1890), der ein bekanntes Buch von ihm rezensierte. ${ }^{2}$ Jhering war ferner Ehrengast an der Feier zum 300-jährigen Gründungsjubiläum der Universität Leiden im Jahr 1875. Nachdem der Professor für römisches Recht an der Universität Leiden, J.E. Goudsmit (18131882), einen Trinkspruch auf Jhering ausgebracht hatte, ergriff der berühmte deutsche Gelehrte das Wort. Er sprach auf Deutsch ("da er mitteilte, dass er Latein nicht so gut beherrsche wie ein Student aus Leiden") und erklärte, "er sei eigentlich ein halber Holländer, nämlich ein Ostfriese". Anschließend bedankte er sich bei der Leidener Akademie im Namen der deutschen Wissenschaft, "die die Universität Leiden geschätzt hat und heute noch schätzt". Schließlich hob er sein Glas auf die Leidener Studenten. ${ }^{3}$

Jherings Werk wurde in den Niederlanden nicht einhellig positiv aufgenommen. Der Professor für römisches Recht in Groningen und später in Leiden, H.L. Drucker (18571917), war ein großer Bewunderer der Ideen Jherings, hauptsächlich aus dessen späteren Werken. Diesem folgend, lehnte Drucker den Vorrang des "Systems" über das wirkliche Leben und des juristischen Begriffshimmels über die Welt der Tatsachen ab. Er kritisierte genau wie Jhering - den "Doktrinarismus", der "unerbittlich auf seine haargenau definierten Begriffe schwört, und mittlerweile die soziale Bedeutung der Rechtsordnung aus dem Auge verliert."4 Der Amsterdamer Rechtsanwalt J.A. Levy (1836-1920) dagegen war ein ausgesprochener Kritiker zahlreicher Ideen aus Jherings Werk. Dies stand auch mit der Tatsache

$1 \quad$ Leitgedanke von Der Zweck im Recht, der auf der Titelseite des ersten Bandes der 4. Auflage ("Erste Ausgabe in volkstümlicher Gestalt") aus dem Jahre 1904 steht.

2 Z.B. S.J. Hingst, "[Bespr. van:] R. von Jhering, Civilrechtsfälle ohne Entscheidungen", Nieuwe Bijdragen 1870, S. 443-445.

3 "Het Academiefeest", Algemeen Handelsblad, 9. Februar 1875, S. 1-2 (Zitate auf S. 2, linke Spalte).

4 H.L. Drucker, Begrip en dogma in de rechtswetenschap, Haarlem: De Erven Bohn 1889, S. 12. Drucker sprach über Jherings "überwältigende" Argumentation in einem der letzten Paragrafen in Geist des römischen Rechts. Siehe auch W.L.P.A. Molengraaff, "Mr. Hendrik Lodewijk Drucker", Rechtsgeleerd Ma- 


\section{C.J.H. JANSEN}

im Zusammenhang, dass Levy die Geltung des rezipierten römischen Rechts völlig ablehnte. Er nannte die Rezeptionsgeschichte eine Leidensgeschichte für "alle westlichen Völker". Jhering hatte der Rechtswissenschaft nach Ansicht von Levy großen Schaden zugefügt, weil er einen derartigen Nachdruck auf den universalistischen Charakter des römischen Rechts - "Frucht einer eitlen Sucht nach Ursprünglichkeit" - legte: "Nun, meiner Ansicht nach ist Iherings Universalitätsgedanke von Kopf bis Fuß a failure, durch und durch falsch. (...) Und es ist wahrlich keine feige Selbstgefälligkeit für meine eigene Auffassung, sondern Interesse an unserer schönen Wissenschaft, wenn ich die Zeichen des Widerstandes, die sich allmählich auch in Deutschland regen, mit Freude begrüße." Levy wies dazu auf das Urteil von Lorenz von Stein (1815-1890), der als Professor für Staatswissenschaften in Wien Jhering als Kollegen aus nächster Nähe miterlebt hatte und der im Vergleich mit einem anderen Buch über den ersten Teil von Zweck im Recht geschrieben hatte: "Es steckt in diesem dünnen Bande mehr wirklicher Inhalt, mehr fruchtbares Denken als in manchem viel gerühmten, anspruchsvollem Werke, wie z. B. Ihering's Zweck im Recht - einem Buche, das in einzelnen Capiteln doch nur wie ein prächtiges Portal mit majestätischer Inschrift aussieht." 5

Ich gehe in diesem Beitrag näher auf die Aufnahme der Ideen aus der Zweck im Recht in den Niederlanden ein. Das Buch erschien in zwei Teilen, nämlich 1877 bzw. 1883. Jhering schrieb im Vorwort des ersten Teiles, dass er dieses Buch schreiben musste, bevor er sich wieder seiner anderen wissenschaftlichen Arbeit widmen konnte. Der Grundgedanke des Buches war einfach: "Der Zweck des Rechts" war "der Schöpfer des gesamten Rechts". Es gab "keinen Rechtssatz, der nicht einem Zweck, d.i. einem praktischen Motiv seinen Ursprung verdankt". Das Thema des Buches war also "der Zweck als weltbildendes Prinzip". Und: "mit derselben Notwendigkeit, mit der sich nach der Darwinschen Theorie die eine Tierart aus der anderen entwickelt, erzeugt sich aus dem einen Rechtszweck der andere (...)."6

Mit Zweck im Recht verließ Jhering den Bereich der Dogmatik des römischen Rechts. Das war dem Verfasser seines Lebensberichts in der niederländischen Zeitung Algemeen Handelsblad nicht entgangen. "Ihering war der Interpret des römischen Rechts. Er war jedoch als Rechtsgelehrter gleichzeitig auch Philosoph. Außer für seine Bücher hatte er auch ein

gazijn 1917, S. 377: "Eine sozialere Auffassung des Rechts, mehr Aufmerksamkeit für die Rechtsprechung und für das wirkliche Leben erschien uns notwendig, um die stets wachsende Verfremdung zwischen Recht und Gesellschaft aufzuhalten." Siehe auch H.L. Drucker, "Scherz und Ernst in der Jurisprudenz", Rechtsgeleerd Magazijn 1885, S. 239-241. Siehe U. Falk, Ein Gelehrter wie Windscheid. Erkundungen auf den Feldern der sogenannten Begriffsjurisprudenz, Frankfurt am Main: Vittorio Klostermann 1989, S. 10-15.

$5 \quad$ J.A. Levy, "Savigny en de historische rechtsbeoefening (In Piam Memoriam)", Nieuwe Bijdragen voor Rechtsgeleerdheid en Wetgeving 1879, S. 236 ff., S. 259 (Zitat), S. 260-261 (Zitat).

6 R. von Jhering, "Vorrede zur ersten Auflage", in: R. von Jhering, Der Zweck im Recht, I, 4. Aufl., Leipzig: Druck und Verlag von Breitkopf und Härtel 1904, S. V (erstes Zitat) und S. X (zweites Zitat). Nils Jansen und Mathias Reimann, "Begriff und Zweck in der Jurisprudenz - Ein Geburtstagblatt für Rudolf von Jhering", Zeitschrift für Europäisches Privatrecht 2018/1, p. 89 e.v.; p. 109 e.v. 
Herz für das Leben, das sich rund um ihn abspielte. ${ }^{77}$ Der Nachruf für B. Windscheid (18171892) in derselben Zeitung einen Monat später enthielt einen Vergleich mit Jhering. "Ihering war mehr theoretisch, während sich Windscheid mehr mit der Ausübung des positiven Rechts beschäftigte."8 Das hatte der Verfasser des Nachrufs richtig beobachtet. Niederländische Handbücher zum bürgerlichen Recht verwiesen nicht oder kaum auf das Werk von Jhering, jedoch mannigfach auf Windscheids Lehrbuch des Pandektenrechts. Der Groninger Professor N.K.F. Land (1840-1903) berief sich zum Beispiel - und das ist lediglich eine bescheidene Stichprobe - im Teil Zakenrecht [Sachenrecht] (1901) aus seiner Handbuchreihe Verklaring van het Burgerlijk Wetboek auf ungefähr 400 Seiten etwa fünfzig Mal auf Windscheids Buch und kein einziges Mal auf Jhering (auch nicht beim Besitz). In Büchern, die sich spezifisch mit dem Begriff des Besitzes auseinandersetzten, erhielten die Betrachtungen Jherings selbstverständlich Aufmerksamkeit. ${ }^{9}$

Zum Schluss dieser Einleitung sei noch bemerkt, dass Jherings Ansichten über den nackten Egoismus des Menschen und die damit zunehmende Anwendung von Gewalt dafür sorgten, dass sich Der Zweck im Recht in politischen Kreisen einer gewissen Beliebtheit erfreute. Das Buch wurde sogar vor den Karren der niederländischen Sozialisten gespannt. "Ihering lehrt also, dass sich das Proletariat - will es zu seinem Recht gelangen - auf den Thron setzen muss, auf dem jetzt die Bourgeoisie sitzt. Und wo ist der Einfaltspinsel, der meint, dass die Bourgeoisie ihren Platz freiwillig der Arbeiterklasse räumen würde?"10 In diesem Beitrag tritt vor allem Jherings Einfluss auf die Ansichten der niederländischen progressiven Liberalen (wie Drucker, aber auch ein Kritiker wie Levy) in den Vordergrund.

\section{Einige Hauptgedanken aus Der Zweck im Recht (1877)}

Der Zweck im Recht ist in seinem Kern ein methodologisches Buch. Jhering brach darin mit der Begriffsjurisprudenz, der juristischen Technik oder der "produktiven" Rechtswissenschaft, die er im zweiten Teil seines Geist des römischen Rechts verteidigt und untersucht

7 "Rudolf Ihering", Algemeen Handelsblad, 21. September 1892, Titelseite.

8 "Bernhard Winscheid", Algemeen Handelsblad, 28. Oktober 1892, Titelseite. Siehe dazu auch das gemeinsame Porträt van Jhering und Windscheid von R. Leonhard, "Ein Nachruf für Jhering und Windscheid", Rechtsgeleerd Magazijn 1893, S. 248 ff.

9 Die Dissertation von J.C. van Oven, De bezitsbescherming en hare functies, Amsterdam: J.H. de Bussy 1905, ist eine lange Diskussion mit zwei bekannten Schriften von Jhering zum Besitz Über den Grund des Besitzesschutzes (1868) und Der Besitzwille (1889). Auch J. van Kan, Bezitsverhoudingen, Amsterdam: J.H. de Bussy 1913, S. 46 ff. ("Kann der Grundgedanke, auf den sich Jherings Lehre stützt, ein brauchbares Kriterium für die Unterscheidung des Besitzes und Inhaberschaft verschaffen?") setzte sich mit der Lehre Jherings ausführlich auseinander. Siehe H.H. Runia, Bescherming van bezit. Een rechtshistorische leerstellige studie naar de wortels en de totstandkomings-geschiedenis van de huidige regeling in art. 3:125 BW, Den Haag: Boomjuridisch 2016, S. 32-33.

10 "Geweld een ,reaktionaire' factor!", Recht voor Allen, 2. und 3. Dezember 1893, Titelseite. Jhering behandelte das Verhältnis zwischen Recht und Gewalt in Der Zweck im Recht, Kapitel VIII, Par. 2 (Der Mensch). 


\section{C.J.H. JANSEN}

hatte. Er wollte einen neuen Ansatz entwickeln, nach dem das Recht studiert werden musste, einen Ansatz, der sich auf Erfahrungen und Wahrnehmungen stützte und nicht einen Ansatz, der am Schreibtisch ausgedacht worden war. Sein neuer Blickwinkel wurde derjenige der Teleologie: "Keine Handlung ohne Zweck". Jede Pflicht und jedes Recht, jedes Rechtsinstitut und jede Rechtsfigur sind aus einem bestimmten Zweck entstanden. Dieser Zweck war mit dem Verhältnis zwischen dem Individuum der Gesellschaft eng verbunden. Oder wie Jhering in Kapitel VI von Der Zweck "Das Leben durch und für andere oder die Gesellschaft" schrieb: "Es gibt kein Menschenleben, das bloß für sich da wäre, jedes ist zugleich der Welt wegen da, (...)." Und konkreter: "Unser ganzes Leben, unser ganzer Verkehr ist in diesem tatsächlichen unjuristischen Sinn eine Gesellschaft: ein Zusammenwirken für gemeinsame Zwecke, bei dem jeder, indem er für andere, auch für sich, und indem er für sich, auch für andere handelt."11 Der menschliche Wille setzt das Räderwerk der Gesellschaft in Bewegung. Dieser menschliche Wille "heißt der Kampf der Interessen, der Gegensätzlichkeit der Bestrebungen, heißt Egoismus, Eigensinn, Widersetzlichkeit, Trägheit, Schwäche, Bosheit, Verbrechen." Zwang ist eine der Weisen, um den menschlichen Willen zu regulieren (neben zum Beispiel der Belohnung). Der Zwang setzt den Staat und das Recht voraus. ${ }^{12}$ Recht ist - nach Jhering - "der Inbegriff der mittels äußeren Zwanges durch die Staatsgewalt gesicherten Lebensbedingungen der Gesellschaft im weitesten Sinne des Wortes." 13

Welchen Preis muss das Individuum laut Jhering bezahlen, um sich an den Vorteilen des Rechts zu beteiligen? Er zeigte dies zum Beispiel anhand des Eigentumsrechts auf. "Die 'Idee' des Eigentums kann nichts mit sich bringen, was mit der 'Idee der Gesellschaft' in Widerspruch steht." Nach Ansicht vieler Juristen und Laien gehörte die "Unumschränktheit der Herrschaft des Eigentümers" zum Wesen des Eigentumsrechts und war laut Jhering "jede Beschränkung desselben mit der Idee des Instituts unvereinbar." "Meiner Ansicht nach ist diese Vorstellung eine grundirrige." ${ }^{14}$ Die Auffassung von Jhering stand im Widerspruch zu derjenigen seines Zeitgenossen Windscheid. Dieser ging von einem unbeschränkten Eigentumsrecht aus. "1) der Eigenthümer darf über die Sache verfügen, wie er will; 2) ein Anderer darf ohne seinen Willen über die Sache nicht verfügen." Nach Windscheids Auffassung war "[d]as Eigenthum als solches Schrankenlos, aber es verträgt Beschränkungen." ${ }^{15}$ Nach Ansicht von Jhering muss also jeder Eigentümer die Beschränkung seines Eigentums durch den Staat im Interesse der Gemeinschaft erlauben. Das war der Preis, den er bezahlen musste. Im Vertragsrecht war der Preis für das Individuum weniger hoch. In der Tierwelt lebte der Stärkere auf Kosten des Schwächeren, so Jhering, aber das war in der Menschenwelt anders: "gesichertes Dasein auch des Schwächsten und Ärmsten neben dem

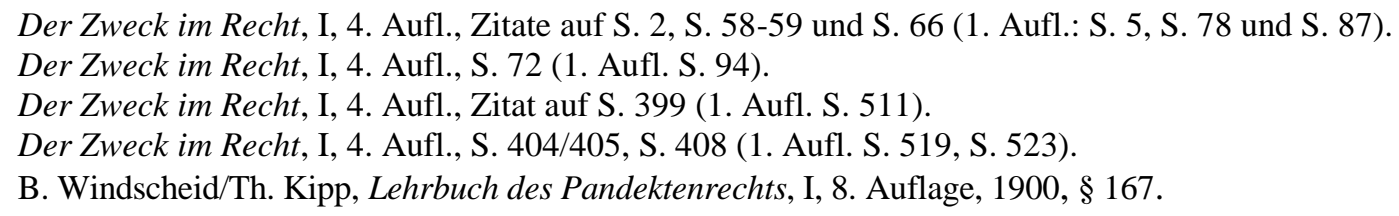


Stärksten und Mächtigsten, das die Gestalt [des Zusammenlebens] in der Menschenwelt." 16 Die wichtigste Triebfeder im vertragsrechtlichen Bereich ist jedoch der Egoismus. Selbst das Handeln zugunsten eines anderen erfolgt aus einem egoistischen Ziel: Das tut jemand, um sich selbst ein gutes Gefühl zu verschaffen und oft erst gegen ein Entgelt. ${ }^{17}$ Jhering räumt den Parteien weitgehende Freiheiten ein: "Das also ist die Logik des zweiseitigen Vertrages: jeder sucht seinen Vorteil und weiß, daß der andere es tut, und das Recht erkennt ihnen diese Befugnis zu, es verstattet dem Egoismus völlig freies Spiel, sofern er sich zur Verfolgung seines Zweckes nur nicht unerlaubter Mittel bedient." ${ }^{18}$ Luig charakterisiert den Standpunkt von Jhering darum auch als liberal (bzw. progressiv-liberal). Jhering widersetzte sich den Auswüchsen des Kapitalismus: Der sozialökonomisch Schwache musste vor dem Starken geschützt werden, und zwar gemäß dem Grundsatz der gleichen Rechte für alle. Jhering war auch Befürworter des Schutzes der Arbeiterklasse, er ging jedoch trotzdem davon aus, dass jedes Individuum seine eigenen Interessen am besten selbst vertreten konnte. Am besten tat es dies, indem es den Interessen der Gesellschaft den Vorrang gab. ${ }^{19}$ Wieacker schrieb, dass er sich anfänglich "als Sohn des klassischen Liberalismus" zu erkennen gab, doch das es sein Verdienst sei, "als einer der ersten Juristen die gesellschaftliche Dimension des Privatrechts erschlossen zu haben, ohne die eine durchdachte Rechtspolitik nicht möglich ist." 20

\section{Der Einfluss von Der Zweck im Recht in den Niederlanden}

Jherings Werk, insbesondere Der Zweck im Recht hat einen großen Einfluss auf das Denken der progressiven Liberalen in den Niederlanden wie die bereits genannten Levy und Drucker ausgeübt. Levy zitierte - trotz seiner Kritik an Jhering als Romanisten - zustimmend aus Der Zweck und war sogar bereit, Jhering für dieses Buch Lob auszusprechen. Er nannte Jhering "von den Juristen einen der ersten". Er wies anschließend auf seine Rechtfertigung der Einschränkung des Privateigentums hin. Jhering betonte den "gesellschaftlichen Charakter der Privatrechte". Levy führte anschließend auf Niederländisch einen bekannten Satz aus Der Zweck an. "Alle Rechte des Privatrechts, wenn sie auch nur das Individuum zum Zweck haben, sind beeinflusst und gebunden durch die Rücksicht auf die Gesellschaft; es gibt kein einziges, bei dem das Subjekt sagen könnte: dies habe ich ausschließlich für mich,

\footnotetext{
16 Der Zweck im Recht, I, 4. Aufl., S. 187 (1. Aufl. S. 241).

17 Der Zweck im Recht, I, 4. Aufl., S. 40 (1. Aufl. S. 53/54).

18 Der Zweck im Recht, I, 4. Aufl., Zitat auf S. 94 (1. Aufl. S. 122/123). Jhering verweist hier auf D. 19,2,23,2. Wie es in der Natur der Dinge liegt, dass beim Kaufen und Verkaufen erlaubt ist, um etwas, was mehr wert ist, für weniger zu kaufen, und was weniger wert ist, für mehr zu verkaufen und einander also auch gegenseitig irrezuführen, so ist dies auch rechtlich zulässig bei Verträgen betreffend das Mieten und Vermieten.

19 K. Luig, "The everlasting universality of Roman Law through the eyes of Rudolph von Jhering (18181892)", Fundamina 1996, S. 19 ff.

20 F. Wieacker, Privatrechtsgeschichte der Neuzeit, Göttingen: Vandenhoeck \& Ruprecht 1967, S. 453.
} 


\section{C.J.H. JANSEN}

'ich bin Herr und Meister über dasselbe', die Konsequenz des Rechtsbegriffs erfordert es, dass die Gesellschaft mich nicht beschränkt." ${ }^{21}$ Auch in Bezug auf den Nutzen und die Notwendigkeit einer gesetzlichen Regelung des Arbeitsvertrags berufen sich progressivliberalen Kreise für deren Begründung auf Jhering. Er hatte in Der Zweck festgestellt, dass Egoismus im Volk und im Staat die Grundlage aller Dinge war. "Der römische Charakter mit seinen Tugenden und Fehlern zusammengefasst ist nichts anderes als ein System des disziplinierten Egoismus... Dem unersättlichen Dämon der Selbstsucht wird alles geopfert: das Glück und das Blut der eigenen Bürger so gut wie die Nationalität fremder Völker. Die Welt, die diesem Dämon ... gehört, ist allen Lebens und seiner schönsten Dinge beraubt. Es ist eine Welt, nicht von Menschen, sondern durch abstrakte Regeln und Vorschriften gesteuert, eine stolze Maschine, bewundernswert durch ihre Stärke, durch die Gleichmäßigkeit und Sicherheit, mit der sie arbeitet, durch die Kraft, die sie entwickelt und mit der sie alles zermalmt, was sich ihr in den Weg stellt -- doch es bleibt eine Maschine: Ihr Meister war zugleich ihr Sklave." Die liberale Idee der Staatsenthaltung wurde jedoch aufgegeben; das allgemeine Interesse trat in den Vordergrund. "Man beginnt einzusehen, dass das Heil der Gemeinschaft in sozialen Gesetzen, die ihre Grundlage in der Gerechtigkeit haben, gefördert werden muss."22

Der Zweck hatte nicht nur einen Einfluss auf (progressiv) liberale Politiker. Der Zweck als der Schöpfer des Rechts übte auch auf niederländische Rechtsphilosophen eine Anziehungskraft aus. Die Rechtsphilosophie geriet im Laufe des 19. Jahrhunderts in Vergessenheit und erlebte in den Niederlanden am Ende des 19. Jahrhunderts ihren Tiefpunkt. G.E. Langemeijer (1903-1990) drückte sich zum "Tod" der Rechtsphilosophie kristallklar aus:

"Man kann jedenfalls gesichert anführen, dass in den Niederlanden zu diesem Zeitpunkt [etwa um 1880] keine Rechtsphilosophie ausgeübt wurde."23

Dank dem rechtsphilosophischen Werk deutscher Juristen wie J. Kohler (1849-1919), R. Stammler (1856-1938) und Jhering nahm das Interesse für das Denken über die Grundlagen des Rechts in den Niederlanden zu. Nach Ansicht des Amsterdamer Professors für Staatsrecht und Rechtsenzyklopädie A.A.H. Struycken (1873-1923) drang in Bezug auf das

21 J.A. Levy, 'De rechtsgrond der ouderdomsrente', Algemeen Handelsblad, 9. April 1916, Morgenblatt, Zweites Blatt, S. 5. Levy verweist auf Der Zweck im Recht, I, S. 415 (1. Aufl. S. 532). Siehe auch J.A. Levy, "De sociale politiek der ,Liberale Unie'", De Tijd, 15. Juli 1895, Titelseite. Levy kritisierte ja gerade den "schändlichen Individualismus des römischen Rechts." Siehe auch J.A. Levy, "Het Romeinsche recht", Algemeen Handelsblad, 3. Dezember 1896, Abendblatt, Zweites Blatt, Titelseite. "De Arbeids-overeenkomst", Nieuwe Tilburgsche Courant, 19. Januari 1899, Titelseite. Derselbe Artikel erschien unter dem Titel "Bescherming van den Arbeid" im Nieuwe Tilburgsche Courant, 9. Januari 1904, Titelseite.

23 G.E. Langemeijer, De wijsbegeerte des rechts en de encyclopaedie der rechtswetenschap sedert 1880, Geschiedenis der Nederlandsche Rechtswetenschap, Deel VI, Amsterdam: Noord-Hollandsche Uitgevers Mij 1963, S. 6. 
"Warum" der verbindenden Kraft von Rechtsregeln immer mehr der Gedanke durch, "dass nur durch die teleologische Schule eine Antwort gegeben werden kann". Struycken schrieb in seiner Dissertation sogar, dass "der größte Teil der juristischen Verfasser - was die Grundlage der Sache betrifft - die Theorie von Ihering zur ihrigen machen". Wie Jhering führte er an, dass Begriffe wie Gut und Böse, Recht und Pflicht die Anerkennung eines zu erstrebenden Zwecks einschlossen. Struycken formulierte einige Anforderungen, denen dieser Zweck genügen musste. Der Zweck musste ein einziger für alle Menschen, alle Zeiten und alle Orte sein. Der Zweck musste überdies "ein Bestimmungszweck sein, der auf das Leben des Menschen gelegt ist, wonach der Mensch sein Leben zu richten hat." Schließlich musste der Zweck ein Endzweck sein, "der letzte Zweck des Menschen auf Erden". Genau in diesem Punkt hatte sich Jhering vergriffen. In Der Zweck im Recht fehlte eine Umschreibung des Endzwecks. "Ihering dringt nicht tief genug durch, weil er sich mit der auf Erfahrung gründenden Aussage 'Recht ist die Sicherung der Lebensbedingungen der Gesellschaft in Form des Zwanges' zufrieden gab, ohne zu untersuchen, was etwas zu einer Lebensbedingung der Gesellschaft macht, noch sich zu fragen, warum diese Lebensbedingungen geschützt werden müssen."24 Übrigens war Struycken vom Tiefgang der Gedanken Jherings nicht sehr beeindruckt. "Dass Iherings Betrachtungen in seinem eigenen Gedankengang oft äußerst oberflächlich sind, kann nicht geleugnet werden." 25

Neben Struycken haben auch andere niederländische Universitätsprofessoren für Rechtsenzyklopädie - wie Struycken auch schon anführte - dem Zweckgedanken Jherings in ihren Vorlesungen oder Schriften einen wichtigen Platz eingeräumt. Ich nenne dabei zwei Namen, nämlich D.G. Rengers Hora Siccama (1876-1962), Professor für Rechtsenzyklopädie in Utrecht und I.B. Cohen (1867-1954), unter anderem Professor für Rechtsenzyklopädie in Groningen. ${ }^{26}$ Cohen verdeutlichte die Wirkung des Zweckgedankens im Recht anhand eines Beispiels aus der Bauwelt, das er in Der Zweck gefunden hatte. Ein Haus ist etwas anderes als eine Gesamtheit von Steinen. "Aber das aus einer Masse von Steinen erbaute Haus ist kein bloßer Plural vom Singular Stein, sondern ein neuer die sämtlichen einzelnen Bestandteile zur Einheit zusammenfassender Gegenstand, eine Einheit, ein Ganzes. Der bestimmende Gedanke, der diese Einheit hergerufen hat, ist der Zweck, das Haus dient einem andern Zweck, als der Stein, und die äußere Veränderung, die mit den Steinen vorgegangen ist, ist

24 Siehe A.A.H. Struycken, Encyclopaedie van het recht (1906-1907), UB Amsterdam VIII F 34*a, S. 2830. Siehe zur Kritik der Tatsache, dass Jhering keinen Endzweck formuliert hatte, auch: A.A.H. Struycken, Het Rechtsbegrip. Theoretische onderzoekingen, Leiden: Eduard IJdo 1903, S. 100-101 (Zitate auf S. 100). Siehe für den Fundort von Jherings Definition von Recht Fn. 12 dieses Beitrags. Struycken verwies auf Thomas von Aquin (1225/1226-1274), der den teleologischen Ansatz ebenfalls befürwortet hatte und einen Endzweck formuliert hatte. Jhering schrieb ehrlich: "Ich meinerseits hätte vielleicht mein ganzes Buch nicht geschrieben, wenn ich sie [die Gedanken von Thomas] gekannt hatte, denn die Grundgedanken, um die es mir zu tun war, finden sich schon bei jenem gewaltigen Denker in vollendeter Klarheit und prägnanter Fassung ausgesprochen." Der Zweck im Recht, 4. Aufl. II, S. 126, Fn. (1. Aufl. S. 162, Fn.). Siehe auch "Katholiek en Socialist", De Tijd, 24. September 1931, Titelseite.

25 Struycken, Het staatsbegrip, S. 101, Fn. 1.

26 C.J.H. Jansen, Van Struycken tot Telders. Een onderzoek naar de Inleiding tot de rechtswetenschap in de eerste helft van de twintigste eeuw, Zwolle: W.E.J. Tjeenk Willink 1992, S. 99. 


\section{C.J.H. JANSEN}

nur die Folge der veränderten Zweckbestimmung."27 Ein anderer Rechtsphilosoph und Professor für Rechtsenzyklopädie, der oft auf das Werk von Jhering zurückgriff, war der Leidener Professor W. van der Vlugt (1853-1928) ${ }^{28}$ Schließlich weise ich auf den Einfluss hin, den Jhering über einen Wiener Schüler, den späteren deutschen Strafrechtsprofessor Fr. von Liszt (1851-1919) auf die Gedankenbilder der modernen Richtung im Strafrecht gehabt hat, der eine sich stark auf die Person des Täters gerichtete Auffassung der Kriminalität befürwortete und das Strafrecht nicht nur als einen Teil der Rechtswissenschaften betrachtete, sondern auch als einen Teil der Sozialwissenschaften. Von Liszts Aufsatz "Der Zweckgedanke im Strafrecht" (1882) hat den niederländischen Professor für Strafrecht und den Begründer der modernen Richtung in den Niederlanden, G.A. van Hamel (1842-1917), tiefgreifend beeinflusst. ${ }^{29}$

Der Zweck im Recht hat schließlich dazu beigetragen, dass eine neue Sichtweise in das Privatrecht Eingang gefunden hat. Sie trat an die Stelle des Legalismus (Gesetzespositivismus), der Auffassung, die die Initiative zur Rechtsentwicklung beim Gesetzgeber niederlegte und stark exegetisch und deduktiv vorging. Die neue Sichtweise entfernte sich ebenfalls von Savignys organischer Rechtsauffassung. Sie nahm "das wirkliche Leben", die Wahrnehmung des Verhaltens der Menschen in der Gesellschaft zur Grundlage. "Bei dieser Methode geht man von der Ansicht aus, dass alle Theorie grau ist, die sich mit der Logik der Tatsachen im Widerspruch befindet. Die Tatsachen bilden auf diese Weise das Material, auf das sich die Untersuchung richtet; man strebt danach, die Tatsachen auf vorurteilsfreie Weise zu sammeln, zu sichten und zu ordnen." ${ }^{30}$ Dadurch wirkte die neue Lehre stark induktiv. Ein ziemlich radikaler Befürworter dieser Auffassung war der Utrechter Professor für bürgerliches Recht H.J. Hamaker (1844-1911). Seiner Ansicht nach bestand zwischen dem niederländischen Bürgerlichen Gesetzbuch und dem bürgerlichen Recht eine Dualität. Studienobjekt ist das gesellschaftliche Leben des Menschen. Das Recht verhielt sich zur

27 Der Zweck im Recht, II, 4. Auflage, Zitat auf S. 139 (1. Aufl. S. 178). I.B. Cohen, Encyclopaedie der rechtswetenschap en Algemeene Rechtsphilosophie, UB Groningen Archief Cohen, S. 51-52. Siehe ferner auch Cohens Rede als Rektor De Dienst der Gerechtigheid (1923).

28 W. van der Vlugt, Algemeene Inleiding tot de Rechtsgeleerdheid, Haarlem: H.D. Tjeenk Willink \& Zoon 1925. Er berief sich hauptsächlich auf Der Geist des römischen Rechts und nannte Jhering "einen berühmten Gelehrten", der gelegentlich die "Unmenschlichkeit" des römischen Rechts "gegeißelt" habe (S. 287). Van der Vlugt berief sich auch in den Beratungen in der zweiten Kammer des niederländischen Parlaments auf die Rechtsauffassung von Jhering: Algemeen Handelsblad, 6. Dezember 1903, Morgenblatt, Zweites Blatt, S. 6.

29 Siehe M.S. Groenhuijsen, D. van der Landen (Red.), De moderne richting in het strafrecht. Theorie, praktijk, latere ontwikkelingen en actuele betekenis, Arnhem: Gouda Quint 1990. Van Hamel ließ sich - neben von Der Zweck im Recht - hauptsächlich von Jherings Der Kampf ums Recht (1872) inspirieren. Er hat dieses Buch 1874 in das Niederländische übersetzt. Siehe die Besprechung van R.T.H.P.L.A. van Boneval Faure, "De strijd om het recht", De Gids 1874, S. 201 ff. Faure nannte die Abhandlung von Jhering genial (S. 225).

30 I. Boer Hz, "Rechtsontwikkeling, theorie en praktijk in de rechtswetenschap", Tijdschrift voor Privaatrecht, Notariaat en Fiscaalrecht (1) 1900, S. 269 ff. (Zitat auf S. 272). Er machte übrigens auch oft Gebrauch von Jherings Kampf ums Recht (1872). 
Gesellschaft wie die Naturgesetze zur Natur. "Das bürgerliche Recht befindet sich nicht im Gesetzbuch, sondern außerhalb, und das Gesetzbuch ist einfach die offizielle Leitlinie, die dem Publikum und der Justiz ausgehändigt wurde, um es kennen zu lernen. Das Verhältnis zwischen den zwei Sachen ist wie das Verhältnis zwischen dem Original und dem Porträt. Das Gesetzbuch ist dessen Abbildung. Ebenso wenig, wie die Tierwelt und das Buch der Zoologie dasselbe sind, verhält es sich mit diesen zwei Sachen." ${ }^{11}$ Andere Vertreter dieser Strömung neben Hamaker waren Drucker und Hamakers Utrechter Kollege für Handelsrecht, W.L.P.A. Molengraaff (1858-1931). ${ }^{32}$ Vor allem Drucker - es sind "deutsche Zustände und deutsche Betrachtungen", hauptsächlich inspiriert durch das Werk von Jhering, insbesondere von Der Zweck und Scherz und Ernst in der Jurisprudenz, woran seine Ausführungen stets anknüpften - ergriff Partei für dessen Bekämpfung der Begriffsjurisprudenz, obwohl er gleichzeitig schrieb: "Was uns betrifft, sollte die Furcht vor schädlicher Begriffsjurisprudenz viel weniger groß zu sein, wir laufen eher Gefahr, der entgegengesetzten französischen Methode [man liest: Die Freirechtsbewegung] zu verfallen." Er rühmte insbesondere das römische Recht und die römischen Juristen um ihre Methode, "die Form, die Technik des Rechts". "Wir lernen die römischen Rechtsgelehrten (iurisconsulti) als unermüdliche vorurteilslose Beobachter des menschlichen Lebens und Arbeitens kennen, als Meister im Finden der Regel, die sich dazu eignet, um neu entstandene Bedürfnisse zu befriedigen." Moderne Juristen haben dieses Beispiel ihrer römischen Vorgänger ignoriert. Sie verloren die Anforderungen der Wirklichkeit aus dem Auge. "Bei den meisten von ihnen [bekleidet] das Dogma die Stelle der unbefangenen Betrachtung der Tatsachen." ${ }^{13}$

\section{Abschließende Bemerkungen}

Die Ideen von Jhering haben in niederländischen Juristenkreisen einen ziemlich großen Widerhall gefunden, insbesondere bei einer Gruppe (liberaler) Juristen, die am Ende des 19. Jahrhunderts gewirkt haben. Sie schufen ein neues wissenschaftliches Klima. Der Ruf nach einer größeren Aufmerksamkeit für das gesellschaftliche Leben und für die Praxis des täglichen Lebens bei der Lösung juristischer Probleme und der Aufruf, soziale Probleme als

31 Siehe insbesondere seine Reden als Rektor Dogmatische en empirische rechtsbeschouwing (1884) und Het recht en de maatschappij (1888), enthalten in: W.L.P.A. Molengraaff und C.W. Star Busmann, Verspreide Geschriften van Mr. H.J. Hamaker, VII (Algemeene rechtsgeleerdheid), Haarlem: De Erven Bohn 1913, S. 1 ff. bzw. S. 19 ff. H.J. Hamaker, Dictaat over het Algemeene Deel van het Burgerlijk Recht (1891-1892) und (1892-1893), UB Utrecht, Hs 16 D 33, S. 8; H.J. Hamaker, Burgerlijk recht, UB Utrecht, Hs B 1 d-I, S. 4.

32 Siehe ausführlich C.J.H. Jansen, "Begriffsjurisprudenz, deductie of inductie. Variaties op een motto ontleend aan R. von Jhering (1818-1892)", RM Themis 1991/2, S. 63 ff. und C.J.H. Jansen, De wetenschappelijke beoefening van het burgerlijke recht in de lange $19^{e}$ eeuw, Deventer: Wolters Kluwer 2015, S. $131 \mathrm{ff}$.

33 Drucker, "Scherz und Ernst in der Jurisprudenz", S. 240-241 und Drucker, Begrip en dogma in de rechtswetenschap, S. 5 (Zitat) und S. 10 (Zitat). 


\section{C.J.H. JANSEN}

juristische Probleme zu betrachten, war in dieser Zeit laut, nicht nur im Privatrecht, sondern auch im Strafrecht. Der Zweck im Recht - der Zweckgedanke im Recht - fiel in den Niederlanden auf fruchtbaren Boden, auch, weil das Interesse für die Grundsätze des Rechts am Ende des 19. Jahrhunderts wieder zunahm. Der Zweck führte in der niederländischen juristischen Debatte eine neue Methode ein. Jhering stand auf diese Weise mit seinem Buch an der Wiege eines "neuen Lebens" der etwas eingeschlafenen Wissenschaft des Privatrechts im 19. Jahrhundert.

Corjo Jansen ${ }^{34}$

Nijmegen

34 C.J.H. Jansen ist Professor für Rechtsgeschichte \& Bürgerliches Recht und Vorsitzender des Forschungszentrums Onderzoekcentrum Onderneming \& Recht (OO\&R) der Radboud Universität in Nijmegen (Niederlande). 